\title{
Variations
}

Variations

Revue internationale de théorie critique

18 | 2013

"La poésie est une arme chargée de futur »

\section{Quelques techniques pour reprendre la parole dans les zones périurbaines pavillonnaires}

\section{Eric Chauvier}

\section{(2) OpenEdition}

Journals

Édition électronique

URL : http://journals.openedition.org/variations/630

DOI : 10.4000/variations. 630

ISSN : 1968-3960

Éditeur

Les amis de Variations

Référence électronique

Eric Chauvier, «Quelques techniques pour reprendre la parole dans les zones périurbaines

pavillonnaires », Variations [En ligne], 18 | 2013, mis en ligne le 31 mai 2013, consulté le 19 avril 2019

URL : http://journals.openedition.org/variations/630 ; DOI : 10.4000/variations.630

Ce document a été généré automatiquement le 19 avril 2019

Les ami•e•s de Variations 


\title{
Quelques techniques pour reprendre la parole dans les zones périurbaines pavillonnaires
}

\author{
Eric Chauvier
}

1 La périurbanisation renvoie à un processus d'extension spatiale de la ville vers la campagne qui prend effet en France à la fin des années 1960. Les conséquences sont plus ou moins remarquables: apport de population active, modification des paysages, implantation de nouveaux modes de vie, accroissement des déplacements pendulaire $»^{1}$. La péri-urbanité que je vais évoquer n'est pas assimilable aux banlieues riches, non plus aux banlieues pauvres. Elle n'est pas même un entre-deux, mais un ailleurs, satellisé dans des zones relevant de l'addition improbable de secteurs pavillonnaires, de prairies, de bois, de friches industrielles, de panneaux publicitaires surdimensionnés, et d'axes routiers reproduisant ad nauseam leurs ronds-points et leurs échangeurs. Même ceux qui n'y vivent pas traversent cette péri-urbanité, mais sans que leur attention ne se fixe sur ses reliefs. On demeure à sa surface. Mais ce ne sont là que des informations superficielles. La principale caractéristique des zones périurbaines pavillonnaires est qu'elles sont épistémologiquement déclassées. Les médias évoquent des «banlieues molles ", mais l'usage de ces mots révèle surtout leur incapacité à désigner des paysages à la fois très disparates et étrangement unifiés. Les urbanistes semblent un peu plus précis au moment de caractériser ces zones, spécifiant quelques peu ces «banlieues molles » en les opposant à d'autres périphéries plus identifiables ("riches» ou "pauvres", « cossues » ou « sensibles »). Ils évoquent aussi des «villes périurbaines» (bel exemple d'oxymore), ou encore une "péri-urbanité ", mais cette catégorie, attachée à un critère strictement géographique, ne semble pas plus précise. Il s'agirait en somme d'une zone autour de la ville, qui n'est donc pas exactement la ville: une ville sur un plan administratif mais non éligible comme telle dans une acceptation classique de la ville. Les urbanistes parlent aussi de "rurbanisation », catégorie qui présente l'avantage d'affiner la définition des zones périurbaines pavillonnaires (que je renommerai «ZPP » par commodité) en croisant les modes de vie avec des fragments de nature. Mais cette autre 
désignation demeure trop flottante pour identifier l'entrecroisement inédit de ces formes d'urbanité et de ruralité. D'une façon générale, les analyses des urbanistes ne disent rien de ceux qui vivent dans les secteurs pavillonnaires.

Ces zones échappent aussi aux anthropologues, ces spécialistes de l'humanité en contexte 2. Leur démarches ne s'applique en effet qu'à des mondes urbains où des groupes humains sont clairement repérables: des migrants, des exclus, la classe ouvrière, la grandebourgeoisie, etc. Or, les modes de vie de la ZPP apparaissent extrêmement standardisées, tant au niveau des transports et de la consommation, que des façons de se rencontrer ou de s'associer, ce qui rend difficile le repérage d'une distinction par classes sociales (les marqueurs de classes sociales sont présents, mais dilués).

Les approches critiques sembleront tout aussi peu soucieuses de constituer un matériau à taille humaine. L'architecte urbaniste David Mangin a, par exemple, approché la vie mécanique de la péri-urbanité pavillonnaire dans un ouvrage important, La ville franchisée, mais son propos critique n'a pas pour projet de cerner le mode de vie périurbains. Quant au livre de Jean-Luc Debry, Le cauchemar pavillonnaire, s'il a valeur de pamphlet contre ces formes d'étalement urbain, il ne se réfère à aucun matériau d'enquête, se contentant d'aligner des sources de seconde-mains censées condamner ce "dépotoir" de civilisation.

4 Pour le dire de façon plus synthétique, ces paradigmes objectivant et globalisant sont presque tous inadéquats ou déconnectées des enjeux anthropologiques des ZPP, lesquelles sont vouées à l'angle mort de la recherche et de la pensée critique alors même qu'environ 20 millions de personnes y résident en 2012. Mon hypothèse est que l'imposition de ces modèles exclusifs de connaissance, en confinant cette majorité silencieuse dans des zones d'éteignoir de la prise de parole, en fait une entité à la fois indéterminée et aisément contrôlable. C'est ce qui guidera ma réflexion, qui sera menée en trois temps. Il s'agira d'abord de se demander pourquoi la prise de parole est difficile dans ces zones (pour preuve, aucun mouvement d'Indignés n'y a jamais vu le jour). Quelques techniques seront ensuite proposées, sous la figure tutélaire de Michel de Certeau, pour déjouer les stratégies totalisantes qui y sont en œuvre. Il sera alors possible, dans un dernier temps, de caractériser la possibilité d'une prise de parole dans les ZPP.

\section{Impossibilité de la prise de parole}

\section{Ne pas parler de politique de façon explicite}

5 Ce 2 décembre, au moment d'enregistrer mes achats à la caisse n³4 de l'hypermarché, dans la file de clients je repère trois hommes qui parlent vivement. Ce sont des cadres de l'aérospatiale. Deux d'entre eux habitent dans mon quartier. Je leur ai parlé, un jour, il y a longtemps. Nous avons échangé des propos banals concernant l'équipe locale de football et les prix du kilo de tomates. Puis j'ai cru bon d'énoncer des lieux communs sur l'état du monde, des pensées "politiques » (autrement dit fabriquées par les médias au sujet de la vie politique) très peu élaborées, que j'ai avancées pour faire consensus, par paresse intellectuelle en définitive. Mais, à ma grande surprise, quelque chose, dans mes propos, les a choqués. Leur regard a biaisé et, ostensiblement, ils n'ont pas répondu. Puis ils ont changé de sujet et sont revenus à leurs tomates. J'ai d'abord pensé qu'ils ne partageaient pas mes idées, mais, avec le recul, leur trouble ne peut en aucune façon s'expliquer de la sorte. L'explication est beaucoup plus édifiante: c'est le fait-même de parler de 
«politique » (avec toutes les réserves faites ici à l'usage de ce mot) qui a provoqué chez eux cet état de trouble. Qui étais-je à leurs yeux ? Probablement un trouble-fête, doublé d'un raseur. Ils m'ont salué avec une froide politesse, lourde de sous-entendus.

Cette expérience n'est pas isolée. Je l'ai vérifiée dans d'autres lieux, devant l'école, à l'hypermarché ou dans des lieux institutionnels (associations sportives ou culturelles). Le plus remarquable est que l'attitude des deux hommes ne concerne pas une opposition d'idées. Il est au demeurant impossible de savoir s'ils sont partisans ou non du président. C'est le fait-même de parler de " politique » qui provoque cet évitement et cette attitude défensive à mon égard. Une dépolitisation est en œuvre, qui réside dans le fait de ne pas parler de sujets politiques, mais en tant que ces sujets permettent aussi de parler de soi et de sa participation à la vie périurbaine. Les thèmes conversationnels «tomates » ou «football» n'interdisent certes pas une dose de plaisir, peut-être même une façon de faire passer sa colère. Mais ils se situent sur un registre de discours qui dénie l'usage d'un lexique politique et, par là, empêche la formulation de problématiques signifiant une participation explicite à la vie de la cité périurbaine.

7 Un extrait d'entretien permet d'affiner l'hypothèse d'une absence de prise de parole politique :

Le collectif: Vous vous plaisez ici?

La femme : Euh, (hésitation), je sais pas ce que ça veut dire, j'y vis, euh... j'y vis plutôt bien, voilà...

8 «Je ne sais pas ce que ça veut dire». Ne pas savoir ce que veut dire "se plaire", «se contenter ", "se satisfaire ». Cette tendance se retrouve souvent dans les façons de parler des périurbains. La question de l'épanouissement personnel est en général prohibée dans les discussions ordinaires. Comme dans la scène des deux hommes qui refusent d'employer un lexique politique explicite, ce qui est proscrit, c'est bien la mise en réflexivité de la condition périurbaine dans l'espace public.

9 L'impossibilité de la prise de parole politique explicite constitue une tendance forte des ZPP. Elle prend tout son sens si l'on observe une absence de centralité vivante, autrement dit de place publique à vocation d'agora. Dans la zone pavillonnaire, les voitures sont garées sur les places publiques. Il n'est pas de lieu ou de situation où pourrait s'exprimer une communication intégrant la possibilité d'émettre des commentaires sur soi afin d'être en prise avec ce qui arrive au quotidien. Les bars permettaient autrefois de telles pratiques, mais il en reste très peu dans la ZPP. Ceux qui restent sont conçus pour s'adonner aux jeux de hasard, soient des lieux où, de façon symbolique (en s'en remettant au déterminisme du hasard) chacun, loin de devenir politiquement actif, tend à subir ce qui arrive.

\section{La vocation fonctionnelle et non réflexive de la communication}

L'absence de prise de parole tient aussi à ce que la zone périurbaine est bâtie sur le principe du tout-fonctionnel. Dans les entretiens réalisés, les personnes vantent timidement l'aspect pratique de cette configuration: les transports en commun, les services pour la petite enfance, la zone marchande, les réseaux automobiles. La communication semble elle-même soumise à cette logique du tout-fonctionnel, comme si le cadre physique de la péri-urbanité imprégnait les façons de parler de chacun. Ainsi, il est notable que les relations que se créent les périurbains viennent de ce qu'ils bâtissent un tissus relationnel de façon institutionnelle, le plus souvent par le biais des 
établissements scolaires où se trouvent leurs enfants et par les associations culturelles ou sportives. Cette observation contredit la possibilité de rencontres spontanées telles qu'on peut les faire dans la ville-centre, perçue a contrario comme un lieu de vie intense, avec ses faisceaux de stimuli culturels ou marchands. C'est tout le jeu du théâtre social qui s'en trouve réduit: la séduction (la drague), la «représentation » et le « rôle» tels que les pense Erving Goffman (1974) ou tels que John Foot Whyte les présente dans ses études sur la ville de Boston (2002). Il faut aussi noter que, d'une façon générale, les périurbains se perçoivent comme les rebuts ou les refoulés de la ville-centre en termes de transport (ici la voiture est hégémonique), d'activité (ici, c'est la cité dortoir) et de choix de consommation (ici c'est l'hypermarché). Il en va aussi de la sorte concernant l'usage de la communication, comme si la configuration urbanistique et humaine des ZPP rendaient improbable la prises de parole réflexive et politique.

\section{L'effacement des marqueurs de classes sociales}

11 Si cette vocation non réflexive de la communication permet de repenser la dépolitisation en œuvre dans le quotidien, elle permet aussi de questionner une autre forme de prise de parole: l'expression de la détresse et, d'une façon générale, de ce qui échappe au caractère programmatique du tout-fonctionnel. Lorsque les personnes ne connaissent pas de problèmes dans leur vie, le déni de réflexivité paraît assez peu importer. Chacun reste dans le confort d'un entre-soi. Mais lorsque des accidents de la vie surgissent, la vie fonctionnelle ne répond plus du tout aux attentes des personnes. Qu'est-ce que devenir pauvre, par exemple après la perte de son emploi, dans une zone où la réflexivité est proscrite ? Il s'agit là d'une toute autre approche du pauvre dans la ville. Il n'est bien sûr plus réductible à l'imagerie du SDF. Il n'est pas non plus réductible à une marque identifiée. Il est avant tout celui qui ne peut exprimer sa détresse psychologique et matérielle dans une zone où tout fonctionne, une zone où prédomine un discours " opérationnaliste "- «les noms des choses indiquant immédiatement leur mode de fonctionnement » (Marcuse : 1973, 111).

Un effet de cette communication est d'effacer les marqueurs de classes sociales. Or, il existe une diversité importante de classes sociales dans les zones pavillonnaires. La disparité s'observe surtout entre des résidents historiques ayant hérité de biens immobiliers et aux revenus plutôt modestes, les cadres et technicien supérieurs (la majorité des résidants) et les nouveaux riches aux villas californiennes qui ont fait fortune dans la location de locaux commerciaux (la poule aux œufs d'or de la ZPP). Cette opacification des marqueurs et des rapports de classes peut être considérée comme un effet de la neutralisation de la communication. Sans ces marqueurs, la prise de parole politique devient en effet plus difficile à diriger et, par là, plus improbable. Riches ou pauvres les périurbains tendent à annuler tous signes identifiant leur appartenance à des classes.

\section{L'impossibilité de transformer la vie ordinaire en contexte}

L'impossible prise de parole des périurbains est liée à la difficulté de promouvoir leur vie quotidienne comme le cadre d'une communication affinée, qui produirait une repolitisation de ce quotidien, en un exercice à la fois critique et réflexif, qui donnerait des outils pour verbaliser le vécu. Ce rapport au contexte est déterminant pour comprendre 
les nouvelles formes de résignation en occident. Les Indignés d'Espagne, de France ou de New York, en se réappropriant la rue, ont aussi tenté de se réapproprier un contexte, celui de la ville, ce lieu ordinaire devenu lieu de souffrance et d'oppression. Leurs revendications portaient sur ce droit fondamental à être de nouveau en prise avec une "réalité" - ce droit que les discours de l'économie ou de la gouvernance mondialisée leur refusent. Or, les ZPP ne peuvent accéder à ce droit à la parole par leur impossibilité à transformer la vie ordinaire en contexte maîtrisable et, par là, en matière politique. Ce n'est pas un hasard si les mouvements des Indignés prennent forme dans les centres des villes. Par leur configuration urbanistique et leu spécificités anthropologiques, ces villes périphériques ne peuvent produire et accueillir de tels mouvements de résistance basés sur la prise de paroles politique. Pourtant, l'importance de ces villes périphériques ne cesse de croître sur un plan démographique, augmentant à mesure la propension à la résignation.

\section{Déjouer les stratégies totalisantes : Michel de Certeau}

14 La perception de l'impossibilité de cette prise de parole m'a conduit à mettre au point un ensemble de techniques ethnographiques adaptées à une double contrainte: sortir de cette vie engoncée pour produire du savoir: identifier et caractériser le caractère déterministe et aliénant de cet engoncement. Sur ce point, l'œuvre de Michel de Certeau s'est révélée tout à fait salutaire en ce qu'elle s'oppose à tous les déterminismes pour leur substituer des outils visant à détourner les représentations, les codes et les usages de la vie quotidienne.

Les déterminismes, Michel de Certeau les désigne comme "stratégies totalisantes", autrement dit « ce qui contraint avec l'apparence du rationnel ». Il leur oppose l'art de la "tactique", soit un «calcul qui ne peut pas compter sur un propre, ni donc sur une frontière qui distingue l'autre comme une totalité visible. La tactique n'a pour lieu que celui de l'autre. Elle s'y insinue, fragmentairement, sans le saisir en son entier, sans pouvoir le tenir à distance. Elle ne dispose pas de base où capitaliser ses avantages, préparer ses expansions et assurer une indépendance par rapport aux circonstances. » (Certeau, 1990, 57)

Depuis sa visite au sommet du World Trade Center, au 110e étage, Michel de Certeau sait que ce panorama impressionnant transforme et met à distance, mais en créant un simulacre, une fiction du savoir, une position surplombante et panoptique, qui permet de voir sans être vu. Certeau préfère quitter cette posture de surplomb pour analyser $l^{\prime}$ ' obscur entrelacs des conduites journalières » et, pour ce faire, arpenter la ville au « ras du sol ». C'est cette tactique que j'ai utilisée pour ethnographier la zone périurbaine pavillonnaire. J'ai consigné un ensemble d'observations selon divers procédés et tenant compte de trois étapes distinctes.

17 La première consiste à déjouer les routines, les habitudes de perception et de cognition à partir de quatre types de procédés :

- Marcher dans une zone où la voiture est hégémonique

- Ecouter plutôt qu'entendre

- Interroger ce qui semble ne pas fonctionner, s'arrêter sur des anomalies qui, en d'autres temps, ne seraient pas interrogées. 
- Créer des expériences de perturbation à la façon des breaching experiences d'Harold Garfinkel. Penser l'étrangeté du monde social. C'est au ras-du-sol que, selon Certeau, se trouve 1' « étrangeté du quotidien », ce qu'il appelle aussi l'« inquiétante familiarité de la ville »3.

\section{Quelques techniques}

\section{Marcher dans la zone dévolue aux voitures}

La marche permet la découverte, dans la parcelle d'un pavillon, d'un panneau publicitaire représentant des cerises rouges et juteuses vendues dans l'hypermarché du coin. Cette technique consiste à quitter la voiture pour changer son point de vue. Cette observation au ras du sol révèle d'abord notre rapport au temps dans des lieux rythmés par ces rappels publicitaires qui suivent les saisons : affiche de chapons à Noël, de cerises au printemps, de tomates en été, etc. Ce temps n'est pas incarné par nos pratiques, soit une définition classique du temps en anthropologie, mais imposé par cet affichage saisonnier. L'effet de standardisation représentationnelle est très marqué, révélant une première stratégie totalisante, celle de la société marchande, qui impose et incorpore ses repères temporels dans le quotidien flottant de l'automobiliste.

L'exemple du panneau publicitaire révèle aussi la satellisation des périurbains autour de l'hypermarché et le fait que l'organisation de la vie se fait essentiellement en son nom et en son pourtour. Les publicitaires de l'hypermarché ont une vision panoptique de la zone périurbaine, la même que Michel de Certeau critique en haut des tours du WTC.

\section{Ecouter plutôt qu'entendre}

Après avoir décidé de sortir de mon pavillon pour écouter le bruit de la rue et ne plus seulement l'entendre, comme un bruit de fond, il ressort que le temps s'égrène au rythme du flux des voitures circulant dans la rue. Notre temps est rythmé de manière quasi inconsciente par les bruits des voitures et ce en fonction des horaires d'ouverture et de fermeture de l'hypermarché.

Ici la technique, des plus simples, consiste à écouter ce qui d'habitude s'entend, imposant une attention soutenue au détriment de la routine quotidienne. Cette technique va permettre de découvrir et de déjouer la stratégie totalisante de la voiture et à son hégémonie, elle-même liée à une autre stratégie, celle de l'hypermarché.

\section{Interroger les dysfonctionnements}

24 Les pavillons ont très souvent des baies vitrées très larges pour laisser entrer la lumière, et aussi pour communier avec des éléments naturels, par exemple un parc arboré. Mais 
un problème se pose : les habitants de notre quartier observent que des oiseaux, surtout des buses, se cognent et s'assomment sur leurs baies vitrées. Lorsqu'ils évoquent cela, ils font surtout part de leur répulsion à l'idée d'avoir à saisir ces corps d'oiseaux morts. Ils mentionnent aussi leur désarroi de ne pas savoir où jeter ces dépouilles. Ils n'osent les déposer dans une de leurs poubelles. Dans celle qui est destinée à trier le "papier », un tel acte est inconcevable; dans la poubelle destinée au tri alimentaire cela leur pose un problème qui les perturbe beaucoup, mais sans parvenir à identifier leur malaise. Voilà l'exemple type d'anomalie destinées à demeurer dans l'angle mort de la vie quotidienne, même si les périurbains ressentent un malaise. Ils en parlent sans mettre de sens dessus : pourtant, cette observation fait du sens.

La technique consiste ici à déplacer son regard et à s'extirper du fonctionnalisme ambiant pour se rendre attentif à ce qui ne fonctionne pas, à savoir le recyclage des déchets. Elle révèle alors le caractère inapproprié de l'habitat pavillonnaire dans cette zone semirurale ainsi qu'un rapport mutilé à la nature. C'est aussi ce qui se traduit dans le tri des déchets. Cette pratique, moderne pas excellence, ne peut surmonter ce problème ponctuel. Autrefois, il n'y a pas si longtemps, les animaux morts étaient enterrés au fond du jardin avec un peu de chaux vive pour éviter les odeurs. Mais nous avons perdu ce savoir-faire. Le mode d'habitat, conçu et validé en amont par les collectivités et par les lotisseurs, constitue une stratégie totalisante que l'on peut identifier par l'usage de la technique du regard décalé.

\section{Créer des expériences de perturbation}

Les breaching experiences, pensée par Harold Garfinkel visent à introduire de la perturbation et de l'étrangeté dans des situations de la vie sociale de façon à révéler des "allant de soi ", autrement dit des comportements adaptatifs révélant par défaut la norme sociale.

Dans la galerie de l'hypermarché, un peu avant Noël, une ferme a été reconstituée spécialement pour les enfants, «la ferme des bébés animaux », avec en sous-titre : "un paradis pour les enfants ». S'y côtoient des chevreaux, des lapereaux, des poussins et des chiots. Une jeune femme avec une chasuble jaune fluorescente s'occupe de ces animaux. Je lui fais part de mon étonnement: comment a-t-on pu ainsi reconstituer une vie animale autour d'un thème, les « bébés animaux » au mépris des liens biologiques avec les parents. Et comment les enfants se représentent-ils désormais ces animaux? Comme leurs jouets? La jeune femme, embarrassée, me montre qu'elle ne peut répondre, puis elle détourne le regard. Autour de moi, le malaise gagne l'ensemble des personnes présentes.

La tactique réside ici dans le fait de questionner ce qui d'habitude ne se questionne plus. Je sens bien que ma question paraît déplacée à la jeune femme qui s'occupe de la crèche. Mais le plus notable est que les personnes présentes ne rétablissent pas tout de suite l'équilibre communicationnel. Or, Goffman a montré que des intéractants sont généralement prompts à sauver la situation et à sanctionner celui qui a causé l'embarras. Il n'est rien de tel ici. Les parents laissent un peu perdurer le malaise, comme si cet interstice de temps laissé à l'anomie pouvait produire du sens - peut-être faire l'objet d'une prise de parole inédite, qui traduirait leur insatisfaction, voire leur colère. Quant au motif du malaise, il peut se référer à la stratégie totalisante liée à cette proposition faite 
aux enfants de se représenter des animaux comme des jouets, et ce dans un but mercantile à la veille de Noël. L'animal est réifié en jouet, en marchandise.

\section{Débusquer les boîtes noires de l'expertise et des médias} ZPP. Il est aussi possible de revenir sur l'usage des mots utilisés par les spécialistes de l'expertise pour qualifier les ZPP. Il en va par exemple du mot " périurbain », utilisé pour les zones pavillonnaires sans plus de spécification. L'adjectif " périurbain» ne désigne rien de précis si ce n'est, étymologiquement, « la périphérie de la ville ». De tels usages du langage renvoient surtout à l'incapacité des scientifiques et des experts à caractériser ces zones dont les pratiques sont tout à fait méconnues. Il faut par extension se demander quel est le sens de ne pas nommer ces zones où vivent 15 millions de personnes? Est-ce une négligence épistémologique? Une façon de contrôler les électeurs périurbains? Référée à la pensée de Michel de Certeau, cette tactique (empruntée à Wittgenstein et à la déconstruction du langage philosophique par l'every day use) permet de déconstruire toutes stratégies totalisantes contenues dans des discours présentés comme savants.

Il en va de même pour ce qui concerne le traitement médiatique de l'information. Ainsi, le journal Télérama consacre en février 2010 un article évoquant, à partir du livre de David Mangin, le monde périurbain pavillonnaire de ce pays en relevant sa « mocheté ». L'usage de l'adjectif «moche" tient à une provocation destinée à remuer les consciences assoupies, trop habituées à vivre au milieu de ces enseignes publicitaires, bâtiments commerciaux informes, couleurs criardes, ronds-points, hypermarchés, etc.

Changer son regard implique ici une activité de négociation entre le lecteur et l'article :

Le lecteur, comme le spectateur, est fondamentalement actif. Il interprète activement un texte en faisant usage de ses compétences singulières et en projetant sur lui divers désirs et attentes. Par ce travail d'interprétation, il donne au texte une signification qui ne préexiste jamais à l'acte de lire mais est toujours comme négociée par lui. (Certeau, 1990, 243)

La lecture offre une tactique de "braconnage " qui permet de mettre en perspective l'usage de l'adjectif «moche». C'est admettre que l'expertise de Télérama n'est qu'une proposition, qui demande encore à être négociée avec des lecteurs. C'est à ce stade que se crée la tactique de résistance: dans la transformation d'une expertise assénée en information soumise à une critique par l'acte de lecture. La question est toujours celle du respect du vécu des périurbains, qui n'est pas à minorer, mais au contraire à élever au rang de repère fiable. Qu'en reste-t-il finalement dans l'expertise ? Cette question amène à considérer le caractère inepte de l'argument esthétique, qui est non seulement incapable de décrire le mode de vie périurbain, mais qui dissuade aussi quiconque d'aller mener la moindre investigation en ce sens. Que reste-t-il, finalement, excepté ce jugement de classe qui fait de la zone périurbaine un monde évaluable à la seule mesure esthétique du point de vue de l'hebdomadaire. Qui sont-ils, ces journalistes centralisés, pour décréter la laideur de la périurbanité ? Qui sont-ils pour porter ce jugement qui, ainsi fait, le rend indigne d'être étudié en tant que tel ?

La consommation, organisée par ce quadrillage expansionniste, ferait figure d'activité moutonnière, progressivement immobilisée et "traitée " grâce à la mobilité croissante des conquérants de l'espace que sont les médias. Fixation des consommateurs et circulation des médias. Aux foules, il resterait seulement la liberté de brouter la ration de simulacres que le système distribue à chacun. Voilà 
précisément l'idée contre laquelle je m'élève: pareille représentation des consommateurs n'est pas recevable (Certeau : 1990, 240).

\section{Ordinaire, dissonance, scepticisme}

A bien des égards, les ZPP pourraient aujourd'hui être considérées comme l'antichambre de l'occident. Le malaise de ces zones périphériques pourrait refléter celui de la civilisation occidentale si l'on admet les stratégies totalisantes qui les caractérisent: standardisation représentationnelle de la société marchande, de la communication sociale, de la circulation, de la consommation, du mode d'habitat, de la nature, de l'expertise et de l'information qui en rendent compte. Prendre la parole implique d'identifier et de caractériser ces stratégies totalisantes et leur fiction de rationalité afin de leur substituer les comptes-rendus de l'expérience vécue par chacun. Pour le dire autrement, c'est dans l'usage-même des techniques mises à jour plus haut que la prise de parole devient possible (aussi dans les techniques qui restent à inventer). Il s'agit en somme de déjouer les routines pour faire émerger du sens, de changer son point de vue sur la vie ordinaire. Mais l'ordinaire n'est pas ici compris dans son sens commun de «banal» ou de « quotidien », mais dans le sens que lui accordent Wittgenstein et Austin : comme une communication qui peut se rompre, comme un lieu d'accord par et dans le langage. Stanley Cavell apporte un prolongement à l'ordinaire en posant la question de la représentativité du locuteur :

- Comment puis-je parler au nom des autres?

- Comment les autres peuvent-il parler en mon nom?

Chaque technique utilisée pour identifier les stratégies totalisantes supposent d'avoir des auteurs, qui sont censés prendre la parole au nom des résidants de la ZPP :

- Comment les programmateurs de la société marchande peuvent-ils parler en leur nom ?

- Comment chacun d'entre nous peut représenter ses contemporains dans une communication sociale stéréotypée?

- Comment les urbanistes peuvent-ils parler au nom des résidants au moment de planifier la circulation et de concevoir le mode d'habitat?

- Comment les experts et les journalistes peuvent-il traduire ce que je vis?

C'est dans le constat de ce déni de représentativité que peut prendre forme l'exigence de prise de parole. Chaque technique renvoie à un porte-à-faux de représentativité entre l'expérience vécue et le métadiscours qui en rend compte. La prise de parole se prend dans cet interstice sceptique, comme une brèche pratiquée dans l'épaisseur de l'ordinaire. C'est aussi admettre que le déni de représentativité est vécu comme une violence, comme une brutalité qui rend nécessaire une action de verbalisation. Ce malaise de civilisation naît du mutisme qui nous est infligé par ceux qui parle à notre place, et en exprimant si mal ce que nous vivons. 


\section{BIBLIOGRAPHIE}

ADORNO Théodor W. , Minima Moralia, Payot,

AUSTIN John, Ecrits philosophiques, Seuil, 1994.

CAVELL Stanley, Les Voix de la Raison, Seuil, 1996.

CHAUVIER Eric, Contre Télérama, Allia, 2011.

DEBRIS Jean-Luc, Le cauchemar pavillonnaire, L'échappée, 2012.

DE CERTEAU Michel, L'invention du quotidien, Gallimard, 1990.

FOOT WHYTE John, Street Corner Society, La découverte, 2002.

GARFINKEL Harold, Recherches en ethnométhodologie, Presses Universitaires de France, 2007.

GOFFMAN Erving, La mise en scène de la vie quotidienne, Minuit, 1973.

MARCUS Herbert, L'homme unidimensionnel, Minuit, 1968.

MANGIN David, La ville franchisée, Ed. La villette, 2004.

WITTGENSTEIN Ludwig, Recherches philosphiques, Blackwell, 1953.

\section{NOTES}

1. Sources : Wikipedia

2. Sur le thème de la ville, il faut bien sûr rappeler les travaux de l'Ecole de Chicago puis, plus tard, ceux de Colette Pétonnet, de Jacques Monod, d'Anne Raulin, de Gérard Althabe, d'Hulf Hannerz, de Michele de la Pradelle, ou plus récemment de Michel Agier et d'Alain Bertho

3. Le passage suivant permet un commentaire plus complet sur l'usage de l'étrangeté dans l'œuvre de M. de Certeau : "D'habitude, l'étrange circule discrètement sous nos rues. Mais il suffit d'une crise pour que, de toutes parts, comme enflé par la crue, il remonte du sous-sol, soulève les couvercles qui fermaient les égouts et envahisse les caves, puis les villes. Que le nocturne débouche brutalement au grand jour, le fait surprend chaque fois. Il révèle pourtant une existence d'en dessous, une résistance interne jamais réduite. Cette force à l'affût s'insinue dans les tensions de la société qu'elle menace. Soudain, elle les aggrave, elle en utilise encore les moyens et les circuits, mais c'est au service d'une «inquiétude » qui vient de plus loin, inattendue; elle brise des clôtures; elle déborde les canalisations sociales; elle s'ouvre des chemins qui laisseront après son passage, quand le flux se sera retiré, un autre paysage et un ordre différent. » (La possession de Loudun, présentée par M. de Certeau, coll. Archives, $n^{\circ} 37$, éd. Julliard, 1970, p. 7) 


\section{RÉSUMÉS}

Les paradigmes objectivants et globalisants des médias, de l'urbanisme et des sciences humaines ne peuvent à ce jour rendre compte de la vie à taille humaine des zones périurbaines pavillonnaires, lesquelles sont vouées à rester dans l'angle mort de la recherche et de la pensée critique alors même qu'environ 20 millions de personnes y résident en 2012. En confinant cette majorité silencieuse dans des zones d'éteignoir de la prise de parole, ces modèles exclusifs de connaissance en font des entités à la fois indéterminées et aisément contrôlables par les pouvoirs médiatiques et politiques. Cette hypothèse guidera notre réflexion, qui s'attachera d'abord à comprendre pourquoi la prise de parole est difficile dans ces zones (par exemple, aucun mouvement d'Indignés n'y a jamais vu le jour). Quelques techniques seront ensuite proposées, sous la figure tutélaire de Michel de Certeau, afin de déjouer les stratégies totalisantes qui y sont en œuvre. Il sera alors possible, dans un dernier temps, de caractériser la possibilité d'une prise de parole dans ces zones épistémologiquement déclassées.

\section{AUTEUR}

\section{ERIC CHAUVIER}

EHESS Paris 